since 2006, persons in working-class mining communities, in addition to those in communities in urban areas, have consumed alarming quantities of opioid medications. ${ }^{4}$ This prompts the question of whether opioid use and subsequent addiction begin in a given patient because of physical injury or whether addiction is the consequence of a collective sense of dejection in the population.

Although it is essential to acknowledge the socioeconomic influences on the physical, men$\mathrm{tal}$, and financial condition of persons in a given population, Bohnert and Ilgen make a compelling argument for exploring these dynamics at the individual level, especially in resource-poor areas, where the need for successful interventions for opioid overdose is substantial. ${ }^{5}$ Given that unique populations require integrated health care approaches, it is imperative for these approaches to be designed with consideration of the complex relationships connecting opioid use, community welfare, and individual social systems.

Kimberly I. McClelland, M.D., M.P.H.

Todd H. Davies, Ph.D.

Joan C. Edwards School of Medicine at Marshall University Huntington, WV

daviest@marshall.edu

No potential conflict of interest relevant to this letter was reported.

1. Bohnert ASB, Ilgen MA. Understanding links among opioid use, overdose, and suicide. N Engl J Med 2019;380:71-9.

2. Case A, Deaton A. Rising morbidity and mortality in midlife among white non-Hispanic Americans in the 21st century. Proc Natl Acad Sci U S A 2015;112:15078-83.

3. Lobao L, Zhou M, Partridge M, Betz M. Poverty, place, and coal employment across Appalachia and the United States in a new economic era. Rural Sociol 2016;81:343-86.

4. U.S. opioid prescribing rate maps. Atlanta: Centers for Disease Control and Prevention, 2018 (https://www.cdc.gov/ drugoverdose/maps/rxrate-maps.html).

5. Voelker R. A day in the life: facing the opioid epidemic in Huntington, West Virginia. JAMA 2018;319:1423-4.

DOI: $10.1056 /$ NEJMc1901540
THE AUTHORS REPLY: We strongly agree with McClelland and Davies that although new population studies continue to provide useful context, ${ }^{1}$ there is a need for individual-level studies of the economic causes of deaths from overdose and by suicide. This is especially true with respect to the study of suicide, since studies of the drivers of recent increases in the incidence of suicide in the United States have been limited. Also, individuallevel analyses would allow for examination of the potential interactions between economic and psychological factors and measures of opioid availability and access.

Although this area of research continues to evolve, the increasing incidence of deaths from overdose and by suicide has resulted in a strong political will for action. It is likely that both individual- and population-level solutions would have benefit. For example, providers could intervene with tailored treatments; concurrently, lawmakers could enact policies that reduce barriers to accessing these treatments and that address economic distress. ${ }^{2}$ These solutions could have synergistic effects to meaningfully decrease the incidence of overdose and suicide.

Amy S.B. Bohnert, Ph.D.

Mark A. Ilgen, Ph.D.

University of Michigan

Ann Arbor, MI

amybohne@med.umich.edu

Since publication of their article, the authors report no further potential conflict of interest.

1. Ghertner R, Groves L. The opioid crisis and economic opportunity: geographic and economic trends. ASPE Research Brief. Washington, DC: Department of Health and Human Services, September 11, 2018 (https://aspe.hhs.gov/system/files/pdf/ 259261/ASPEEconomicOpportunityOpioidCrisis.pdf).

2. Dasgupta N, Beletsky L, Ciccarone D. Opioid crisis: no easy fix to its social and economic determinants. Am J Public Health 2018;108:182-6.

DOI: $10.1056 /$ NEJMc1901540

\title{
Cancer Survivorship
}

TO THE EDITOR: In his review article, Shapiro (Dec. 20 issue $)^{1}$ describes the physical and psychological effects of cancer survivorship. We were surprised by the limited attention paid to important concerns of cancer survivors and their families such as maintaining employment, having access to affordable health care, and managing financial hardship because of medical expenses., ${ }^{2,3}$ These concerns are especially common in the United States.

As shown in Figure 1, cancer survivors are more likely to be unable to work or to be limited 


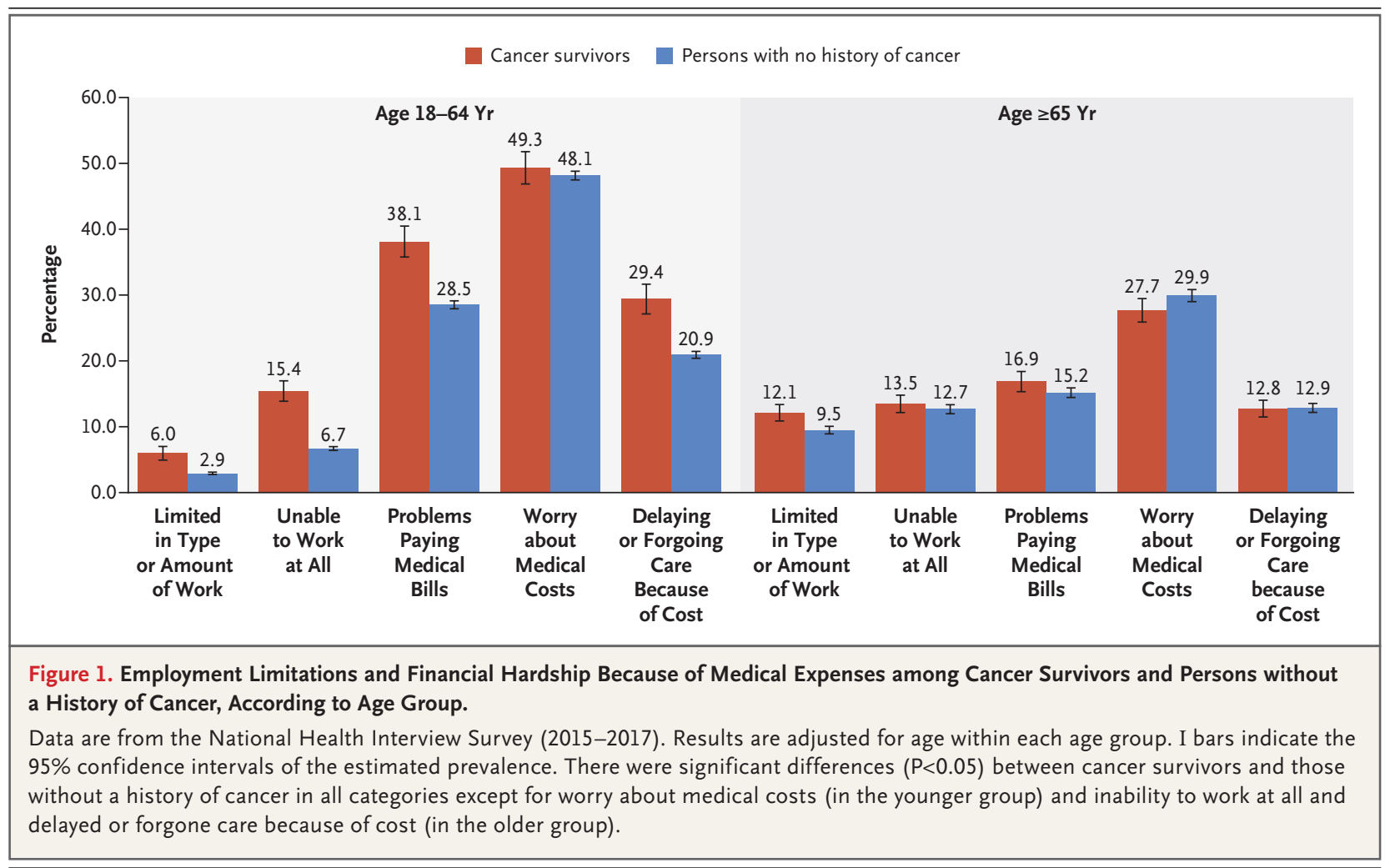

in the type or amount of work that they can do than persons without a history of cancer. Employment limitations can reduce income and access to employer-sponsored health insurance. Cancer survivors are also more likely than persons without a history of cancer to report financial hardship because of medical expenses, including problems paying medical bills, worry related to medical costs, and delaying or forgoing health care because of cost. Cancer survivors who are 18 to 64 years of age (who are not eligible for Medicare coverage because of their age) are most vulnerable. Emerging research suggests that financial hardship is associated with adverse health outcomes. ${ }^{4}$ With increasing health care costs and patient cost-sharing (among the insured), ${ }^{5}$ greater attention to employment limitations, access to care, and financial hardship will be important for all clinicians who care for cancer survivors.

\section{K. Robin Yabroff, Ph.D. \\ Zhiyuan Zheng, Ph.D. \\ Xuesong Han, Ph.D.}

American Cancer Society

Atlanta, GA

robin.yabroff@cancer.org
No potential conflict of interest relevant to this letter was reported.

1. Shapiro CL. Cancer survivorship. N Engl J Med 2018;379: 2438-50.

2. Yabroff KR, Zhao J, Zheng Z, Rai A, Han X. Medical financial hardship among cancer survivors in the United States: What do we know? What do we need to know? Cancer Epidemiol Biomarkers Prev 2018;27:1389-97.

3. Zheng $Z$, Jemal A, Han X, et al. Medical financial hardship among cancer survivors in the United States. Cancer 2019 January 21 (Epub ahead of print).

4. Ramsey SD, Bansal A, Fedorenko CR, et al. Financial insolvency as a risk factor for early mortality among patients with cancer. J Clin Oncol 2016;34:980-6.

5. Kaiser Family Foundation. Payments for cost sharing increasing rapidly over time. October 5, 2017 (https://www.kff.org/ health-costs/issue-brief/payments-for-cost-sharing-increasing -rapidly-over-time/).

DOI: $10.1056 / N E J M c 1900709$

TO THE EDITOR: Although less than half of patients with cancer a decade ago were long-term survivors, now more than two thirds live more than 5 years. As a result, many are affected by numerous long-term complications. Shapiro does not mention that chronic kidney disease, an established but underrecognized complication in cancer survivors, ultimately affects their survival. 
The prevalence of chronic kidney disease is high among cancer survivors; chronic kidney disease of stages 3 to 5 (glomerular filtration rate, $<60 \mathrm{ml}$ per minute per $1.73 \mathrm{~m}^{2}$ of body-surface area) is detected in 12 to $25 \%$ of these patients. ${ }^{1-3}$ This rate is substantially higher than that in the general population. Several studies have established that chronic kidney disease is independently associated with both overall mortality and cancer-specific mortality among patients with cancer, ${ }^{3,4}$ and the greatest risk is incurred by patients who have chronic kidney disease and breast and urinary tract cancers. ${ }^{4}$ Thus, cancer survivors should be monitored for kidney dysfunction, since this common complication is associated with reduced survival.

Ben Sprangers, M.D., Ph.D.

University Hospitals Leuven

Leuven, Belgium

ben.sprangers@uzleuven.be

Kenar D. Jhaveri, M.D.

Zucker School of Medicine at Hofstra/Northwell Hempstead, NY

Mark A. Perazella, M.D.

Yale University School of Medicine

New Haven, CT

No potential conflict of interest relevant to this letter was reported.

1. Launay-Vacher V, Oudard S, Janus N, et al. Prevalence of renal insufficiency in cancer patients and implications for anticancer drug management: the renal insufficiency and anticancer medications (IRMA) study. Cancer 2007;110:1376-84.

2. Janus N, Launay-Vacher V, Byloos E, et al. Cancer and renal insufficiency results of the BIRMA study. Br J Cancer 2010;103: 1815-21.
3. Nakamura Y, Tsuchiya K, Nitta K, Ando M. Prevalence of anemia and chronic kidney disease in cancer patients: clinical significance for 1-year mortality. Nihon Jinzo Gakkai Shi 2011; 53:38-45. (In Japanese.)

4. Iff S, Craig JC, Turner R, et al. Reduced estimated GFR and cancer mortality. Am J Kidney Dis 2014;63:23-30.

DOI: 10.1056/NEJMc1900709

THE AUTHOR REPLIES: Yabroff and colleagues raise an important point concerning the financial burdens of cancer survivors, family members, and caretakers. Sprangers et al. point out that chronic kidney disease is common in cancer survivors. The causes of chronic kidney disease are many and include underlying coexisting conditions (e.g., diabetes and hypertension), direct extension of the cancer to the genitourinary tract, nephrotoxic treatments or medications for cancer, and the lack of dose reductions when nephrotoxic drugs are administered to cancer survivors who have underlying acute or chronic kidney disease. The increased mortality associated with chronic kidney disease is not specific to cancer survivors, since noncancer populations with chronic kidney disease also have increased mortality. ${ }^{1}$

Charles L. Shapiro, M.D.

Icahn School of Medicine at Mount Sinai Uptown

New York, NY

charles.shapiro@mssm.edu

Since publication of his article, the author reports no further potential conflict of interest.

1. Tonelli M, Wiebe N, Culleton B, et al. Chronic kidney disease and mortality risk: a systematic review. J Am Soc Nephrol 2006; $17: 2034-47$

DOI: 10.1056/NEJMc1900709

\section{Case 40-2018: A Woman with Recurrent Sinusitis, Cough, and Bronchiectasis}

TO THE EDITOR: The Case Record by Mojica et al. (Dec. 27 issue) ${ }^{1}$ highlights the importance of considering the diagnosis of cystic fibrosis in adults. We reviewed 842 cases of cystic fibrosis in our electronic health record (EHR) and found that $8.4 \%$ were diagnosed in patients older than 30 years of age. Among 368 patients who received a diagnosis of cystic fibrosis at Vanderbilt University Medical Center, the age at diagnosis has increased over time, including one woman who recently received a diagnosis at 80 years of age.
Case presentation and genetics varied according to age, a finding consistent with other reports. ${ }^{2,3}$ Pulmonary symptoms predominated in patients older than 30 years of age, of whom $91 \%$ did not have the $\triangle$ F508 CFTR mutation. Meconium ileus and failure to thrive predominated in newborns, of whom $71 \%$ were homozygous for the $\Delta \mathrm{F} 508$ mutation.

Diagnosing cystic fibrosis in adults can be difficult. We have proposed that combining multiple EHR phenotypes into a "phenotype risk 